\title{
Patrones de Inserción de las Empresas Multinacionales en la Formación de Competencias Tecnológicas de Países Seguidores*
}

\author{
Ana Urraca Ruiz \\ Professora Adjunta no Departamento de Economia da Universidade Federal Fluminense - UFF
}

\section{RESUMO}

Utilizando base de dados de patentes depositadas no Escritório Europeu de Patentes este trabalho tem como objetivo identificar a existência de padrōes de inserção tecnológica dos países retardatários latino-americanos e asiáticos; demarcar o papel exercido pelas EMN na formação de competências desses países e na aceleração dos processos de captura. $\mathrm{O}$ trabalho conclui que: (i) existem diferentes padrōes de inserção das EMN (empresas multinacionais) de acordo com seu grau de inserção na composição da estrutura tecnológica do país receptor e com a região geográfica deste país; (ii) não necessariamente as EMN duplicam as competências adquiridas em seus países de origem, podendo também aproveitar competências e especialização local; e (iii) existem padrões diferentes entre AL e Ásia, ou seja, a ação das EMN favorece a diversificação da base técnica na Índia e em Hong Kong, enquanto no Brasil e na Argentina as EMN colaboram para sua maior especialização.

\footnotetext{
* La autora agradece a las alumnas Camila Chaves y a Cynthia Bastos, del programa PIBIC-CNPQ, por su inestimable ayuda en la composición de la base de datos que permitió la elaboración de este trabajo y a los profesores Maria Castillo (UFFO, C. Frederico Rocha (UFRJ) y a tres pareceristas anónimos por sus sugerencias y comentarios, eximiéndo a todos ellos de cualquier responsabilidad sobre los resultados aquí presentados.
} 
Palavras-Chave | Competências Tecnológicas; Convergência; Especialização Tecnológica; Países Seguidores; Empresas Multinacionais

Códigos JEL | F23, O14, O31

\begin{abstract}
Using a database composed by patents filed in the European Patent Office, this paper aims to: $(i)$ identify patterns of technological specialization of selected countries from Latin America and Asia; (ii) classify the role played by MNE (multinational enterprises) in the building of technological competencies of these countries as well as their role in the catching-up process. The paper concludes that: $(i)$ there are different patterns of specialization of MNE according to their level of participation in the technological structure of the host country and to the geographic region where the country is located; (ii) MNE do not necessarily duplicate their homebased competencies, but may explore local specialization; and (iii) MNE are more likely to favor diversification of technical base in Asian countries like India and Hong Kong and to deepen specialization in Latin American countries, such as Brazil and Argentina.
\end{abstract}

KeYWORds | Technological Competences; Convergence, Technological Specialisation, Follower Countries, Multinational Enterprises

JEL-CODES I F23, O14, O31 


\section{Introducción}

Las competencias tecnológicas nacionales configuran la especialización tecnológica de un país. Las diferencias observadas en la especialización tecnológica entre países se deben a cuatro factores eminentemente endógenos (Malerba \& Montobbio, 2003): (i) la estructura de la actividad innovadora, que define la trayectoria del desarrollo tecnológico sobre determinada base técnica y específicas formas de innovación; (ii) los Sistemas Nacionales de Innovación (SNI), esto es, la capacidad de innovar de los agentes envueltos en los procesos de innovación (empresas nacionales y multinacionales, universidades y centros públicos de investigación, investigadores independientes, agencias de gobierno, etc.), la articulación entre ellos y la composición de políticas científicas y tecnológicas; (iii) la especificidad del desarrollo de competencias tecnológicas en la firma; y (iv), los vínculos de conocimiento entre tecnologías (spillovers).

Además de estos cuatro factores, la diferente especialización tecnológica entre países no es independiente de la fortaleza de su base técnica. Los países líderes cuentan con una mayor diversificación de sus competencias, mientras que los países seguidores (imitadores, capturadores, o catching-up) cuentan con una especialización tecnológica más concentrada en un escaso número de áreas técnicas (Pianta \& Meliciani, 1996).

Establecido el concepto de especialización tecnológica, la "convergencia tecnológica" puede ser redefinida como un proceso mediante el cual los países tienden a registrar mayores similaridades en la distribución de sus competencias tecnológicas, y no apenas como un proceso que lleva a los países a aproximar sus niveles de desarrollo tecnológico.

Cuando la convergencia tecnológica es entendida en términos de "nivel de desarrollo tecnológico", algunas abordajes más ortodoxas, basadas en las teorías de la convergencia en el crecimiento económico, prevén que los países deberán convergir tecnológicamente casi inercialmente, puesto que existen determinados factores que aceleran el crecimiento de países atrasados y ralentizan el crecimiento de países avanzados. Las teorías del catch-up tecnológico prevén que los países con inferiores niveles tecnológicos, a partir de 
una estrategia "seguidora”, podrían capturar los avances tecnológicos conseguidos por los países líderes, por lo cual, en el largo plazo, se producirá convergencia tecnológica y económica entre ellos.

Sin embargo, la lógica de la dinámica del sistema de acumulación de capital y de conocimiento para el crecimiento puede llevar a resultados opuestos, llegando incluso a aumentar los diferenciales tecnológicos entre países. Los países seguidores, mediante la mera importación de tecnología, apenas serían capaces de capturar tecnologías y conocimiento que se encuentran en fases maduras en el periodo de difusión de la innovación, creando ventajas competitivas en industrias maduras y construyendo competencias sobre tecnologías también maduras, lo que sometería su crecimiento a un patrón de desarrollo de bajo ritmo. Pérez y Soete (1988) destacaron que un proceso real de captura sólo puede ser logrado a través de la adquisición de capacidad para participar en la generación y mejora de tecnologías y no exclusivamente su simple uso. En concreto, los países seguidores conseguirán converger y disminuir sus diferenciales tecnológicos si a través de su sistema nacional de innovación, son capaces de extender su base científica y tecnológica y viabilizar políticas de absorción y de adaptación de conocimiento para poder disminuir los tiempos de entrada, adaptar las tecnologías producidas por los países líderes a las competencias productivas internas y aprovechar las oportunidades que surgen cuando un nuevo paradigma tecnológico emerge.

Por tanto, la convergencia tecnológica pasa en alguna medida por la diversificación de la base técnica, de forma que un proceso real de convergencia significa no sólo aproximación de los niveles de desarrollo tecnológico, sino también aproximación de la distribución de competencias tecnológicas, esto es, de los patrones de especialización tecnológica entre líderes y seguidores.

Bajo estas consideraciones, este trabajo pretende: (i) contrastar si las EMN ejercen un papel activo en la formación de competencias tecnológicas nacionales de países seguidores; (ii) identificar la existencia de diferentes patrones de inserción de las EMN en éstos, concretamente, para un conjunto de países latinoamericanos y asiáticos; y (iii) verificar si, de acuerdo con cada forma de inserción, el papel del las EMN es relevante para acelerar los procesos de captura.

El artículo se divide en cuatro partes. La primera expone las principales hipótesis que la literatura levanta sobre las formas de inserción de las EMN en 
países receptores (sean estos seguidores o no) de acuerdo con sus estrategias de internacionalización de I+D (Investigación y Desarrollo). La segunda y la tercera describen, respectivamente, las fuentes de información y los indicadores utilizados. Finalmente, la cuarta parte analiza los resultados obtenidos.

\section{Estrategias de internacionalización de I+D}

Los argumentos que justifican la internacionalización de la $\mathrm{I}+\mathrm{D}$ en virtud de las ventajas tecnológicas del país receptor avanzan tratando de identificar la fuente de dicha ventaja, generalmente asociada a la trayectoria que sigue el cambio técnico en ellos. En este sentido, la naturaleza de la ventaja tecnológica puede ser "technology push" cuando es relativa a la oferta tecnológica del país en términos de centros de excelencia, alta calificación de científicos e ingenieros o facilidad de acceso a recursos de financiación de la I+D (v.g. biotecnología y semiconductores en los Estados Unidos) (véase Wortmann, 1990); o "demand pull" si la ventaja tecnológica se encuentra en las características de la demanda sobre la especificación de los productos y para el establecimiento de los imperativos tecnológicos (v.g. Japón en productos LCD) (Gerybadze \& Reger, 1999).

La internacionalización de la I+D en virtud de las ventajas tecnológicas de la firma adquiridas en su país de origen surge, desde la teoría penrosiana de la firma, como una extensión de la combinación específica de sus competencias productivas y tecnológicas, tanto si el objetivo perseguido en el exterior es explotar ventajas creadas en casa (home base exploting) como si trata de añadir nuevas técnicas y conocimientos a su conocimiento base para asegurar su supervivencia en el largo plazo (home base aumenting) (Kuemmerle, 1996). Cabe por tanto pensar en dos tipos de esfuerzos realizados en el exterior: (1) explotación de la base técnica desarrollada en el país de origen con esfuerzos de adaptación, llamada de estrategia "duplicativa" (Zander. 1998); y (2) búsqueda de activos complementarios para identificar líneas de crecimiento futuras a través de la extensión de la actual línea de productos, en cuyo caso se denomina "especializado" o "diversificado". La I+D duplicativa suele ser de carácter aplicado adaptado a las necesidades de los usuarios en el país hospedero, mientras que la especialización en determinadas competencias suele estar 
asociada a la búsqueda de conocimiento genérico (básico) con altas posibilidades de aplicación.

De acuerdo con las causas que determinan la internacionalización de la I $+\mathrm{D}$ es posible identificar: $(i)$ patrones geográficos de diversificación vs. duplicación de esfuerzos, si la EMN explota una ventaja tecnológica del país de destino (Cantwell \& Janne, 1999; Gerybadze \& Reger, 1999); (ii) patrones de diversificación vs. duplicación de competencias, si la EMN trata de explotar ventajas tecnológicas propias o crear nuevas competencias (Zander, 1999); o, (iii) patrones geográficos de diversificación vs. duplicación, si la internacionalización es un efecto conjunto de ambas (Patel \& Vega, 1999).

La literatura sobre los aspectos organizativos de la producción internacional de la $\mathrm{I}+\mathrm{D}$ trata de identificar tipos organizativos de acuerdo con las competencias tecnológicas de la EMN, con las particularidades del país de destino y con las características que el cambio técnico adquiere en el sector al que la firma pertenece. Cabe, pues, identificar diferentes tipos de organización de la $\mathrm{I}+\mathrm{D}$ de acuerdo con el tipo de conocimiento base de la EMN, determinadas por su principal sector de actividad, con las características de los productos que desarrolla (su grado de diversificación y diferenciación); con el tipo de I+D que se pretende desarrollar; ${ }^{1} y$, con el lugar en donde se pretenden rentabilizar los resultados de la innovación.

Recientemente, algunos nuevos fenómenos llevaron a pensar si el modelo tradicional centro-periferia seria un buen modelo para explicar eficazmente la naturaleza de las estrategias de internacionalización de I+D de las EMN. Entre éstos, Gerybadze y Reger (1999) destacan los siguientes: (i) la aparición de nuevos centros de excelencia localizados en países fuera de los Estados Unidos que suministraban conocimiento relevante; (ii) la cada vez mayor necesidad de interacción entre actividades productivas, innovadoras y de comercialización, lo cual ha debilitado el papel de la I+D básica desvinculada de otras actividades desarrolladas por la firma; (iii) la dificultad de financiar grandes proyectos de I+D básica, lo que hace más atractivo dispersar los esfuerzos en $\mathrm{I}+\mathrm{D}$ en diferentes centros que desarrollen $\mathrm{I}+\mathrm{D}$ de carácter más

1 Los tipos posibles de I+D que pueden ser desarrollados son dos. Uno relativo a la búsqueda de conocimiento básico de amplia aplicación. El segundo, de carácter adaptativo, surge de acuerdo con las necesidades de los usuarios en los países de destino que permita aprovechar una ventaja local. 
aplicado a fin de conseguir un mayor grado de rentabilización de los resultados; (iv) la conveniencia de intervenir en mercados que siguen ritmos y direcciones de progreso técnico (trayectorias) diferentes en diferentes países; $(v)$ la posibilidad de decidir los imperativos tecnológicos y estándares donde existe falta de regulación; ( $v i)$ la necesidad de conocer las estrategias de las rivales; y (vii) las posibilidades de transferencia de conocimiento a bajo coste que se desprenden de los avances registrados en las tecnologías de la información y de la telecomunicación, que permiten disminuir los costes de transferencia de conocimiento entre unidades aunque estén muy distantes unas de otras. Todos estos nuevos acontecimientos permitieron concluir a los autores con que la organización de la I+D trasnacional se conducía más sobre un modelo descentralizado (ibidem, 1999).

La mayor parte de la literatura asocia la idea de descentralización a la de red aunque no siempre esta asociación es biunívoca. El concepto de organización en red no está claramente definido, siendo un concepto amplio asociado a la observación de múltiples unidades generadoras de innovaciones que actúan con una cierta independencia de la casa matriz en definir sus propias líneas de investigación. El tipo de I+D desarrollado es, cada vez más, de carácter aplicado, aunque no siempre es adaptativo, y los resultados obtenidos pueden ser aprovechados por cada unidad generadora de conocimiento (local for local) y compartidos por la corporación (local for global), siendo esta su principal característica. Esto exige sistemas de gestión que posibiliten la captura de innovaciones en las subsidiarias y superen las barreras de comunicación impuestas por las distancias culturales y geográficas (Zander, 1998).

La composición de las ventajas tecnológicas locales junto con la orientación de las diversas formas organizativas de la I+D trasnacional determinarán diferentes formas de inserción de las EMN en los países hospederos. En primer lugar, en países seguidores, por la debilidad de su base técnica, las EMN tenderán más a realizar I+D adaptativo (aplicado o de desarrollo) siguiendo la orientación de "demand pull" sobre la de "technology push".

En segundo lugar, el objeto de la internacionalización deberá estar más asociado a la explotación de una ventaja adquirida en casa que a la explotación de ventajas locales, esto es, las EMN deberán actuar más sobre la lógica de la "duplicación" que de la "especialización” de competencias originarias. Algunas 
excepciones previsibles serían aquellos casos en los que el país receptor asume liderazgos en tecnologías específicas, en cuyo caso la $\mathrm{I}+\mathrm{D}$ trasnacional puede orientarse a la captura de este tipo de ventajas.

Los efectos sobre el país hospedero pueden ser de diversa naturaleza. En lo que respecta a la convergencia tecnológica, en la línea de argumentación de Pérez y Soete (1988), cabe pensar que si las EMN son capaces de extender la base técnica de los países seguidores hacia campos técnicos más dinámicos y permeables, ayudarán a la reducción de las distancias con los países líderes. Si por el contrario se concentran en explotar ventajas locales, se podría producir un efecto perverso que llevaría a acentuar las especializaciones de los países seguidores en tecnologías poco dinámicas, retardando así el proceso de convergencia.

De comprobarse esta hipótesis, el aprovechamiento de los flujos tecnológicos mantenidos entre países seguidores y EMN dependerá en gran parte de la creación de fortalezas tecnológicas en campos tecnológicos más permeables que abran hacia un modelo de inserción tecnológica más activo (v.g. a través de cooperaciones), lo que, a su vez, permitiría la creación de un círculo virtuoso que aceleraría el proceso de captura y de convergencia con los países líderes.

\section{Bases de datos e indicadores utilizados}

3.1 Los datos de patentes de la European Patent Office (EPO).

A pesar de los inconvenientes de utilizar patentes como indicador de actividad tecnológica (Pavitt, 1988), éstas resultan ser bastantes sólidas para realizar comparaciones internacionales por su elevada homogeneidad, porque abarcan largos periodos de tiempo y porque permiten clasificar resultados de innovación por campo técnico hasta un elevado grado de desagregación de acuerdo con la Clasificación Internacional de Patentes. Por esta última razón, las estadísticas de patentes son las más utilizadas para medir competencias tecnológicas, tanto en el nivel de la firma como en el nivel nacional (Patel e Pavitt, 1993).

La base de datos utilizada en este trabajo es la relativa a patentes depositadas en la European Patent Office (EPO) entre 1978 y 2003 cuyos inventores 
eran residentes en dos diferentes grupos de países seguidores. De un lado, América Latina, incluyendo Argentina, Brasil, Chile, Colombia, Cuba, Méjico, El Salvador y Venezuela. De otro, Asia, que incluye China, Corea del Sur, Hong-Kong, India, Singapur y Taiwán. La selección de países fue realizada de acuerdo con el criterio de número de patentes considerando la desagregación por periodos y por número de campos técnicos. Fueron desestimados los países con menos de 60 patentes para el total del periodo considerado.

La nacionalidad de la patentes viene determinada por la nacionalidad del inventor que participó en su elaboración, puesto que la residencia del inventor indica donde está localizada la competencia tecnológica. El depositante indica si la patente está siendo explotada por una EMN. Se consideró EMN aquella empresa depositante cuyo país de residencia era diferente al país de la nacionalidad de la patente, puesto que esto significa que la firma está internacionalizando su actividad de I+D, esto es, está usando recursos tecnológicos fuera de su país de origen. En este sentido, el carácter de "multinacional" está siendo definido por la medida en que una firma internacionaliza los recursos tecnológicos que utiliza para producir esa patente en concreto. También fue considerada como EMN aquella empresa que, siendo residente en el mismo país que representa la nacionalidad de la patente, estaba vinculada a grupos transnacionales. ${ }^{2}$

La European Patent Office es considerada una base de datos adecuada, en relación a otras bases de datos de patentes, por tres motivos. En primer lugar porque debido a la proximidad y amplitud del mercado de sus países miembros (26 en total), la EPO representa casi automáticamente una segunda opción para países no europeos. En segundo lugar, porque el grado de internacionalización de las actividades de $\mathrm{I}+\mathrm{D}$ que lleva a la generación de las patentes es mucho mayor cuando se utiliza la EPO. De acuerdo con Patel (1995), el análisis de la actividad patentadora de aproximadamente $250 \mathrm{em}$ presas americanas entre 1985 y 1990 en la Oficina Americana de Patentes (USPTO), mostró que apenas el 7,8\% de las patentes totales fue debido a los esfuerzos innovadores de inventores residentes en el exterior y Cantwell (1995), para el periodo 1969-1990, mostró que sólo un 6,8\% de las patentes de

2 Para esto fue realizada uma búsqueda en inter-net a través de los sites de las empresas donde ellas reconocen su pertenencia a grupos transnacionales y la nacionalidad de su capital. 
empresas americanas depositadas en la USPTO eran realizadas por inventores no residentes en los Estados Unidos. Por el contrario, el análisis de Rocha y Urraca (2002) sobre las patentes depositadas en la EPO por una muestra de 116 empresas americanas entre noviembre de 1986 y enero de 1999, mostró que el 21,8\% del total de las patentes registradas tenía inventores no residentes en los Estados Unidos.

En tercer lugar, el coste de registro de una patente en la EPO es relativamente elevado cuando se compara con otras oficinas de patentes, lo que representa un filtro tanto para inventores europeos como de otras nacionalidades y, por tanto, debe proveer estadísticas más homogéneas cuando se pretenden realizar comparaciones internacionales.

\subsection{Indicadores de especialización y convergencia}

La especialización tecnológica se mide a través de la ventaja tecnológica revelada (VTR). Este indicador permite detectar fortalezas tecnológicas o aquellas áreas técnicas donde los países realizan un esfuerzo en patentar superior a la media mundial. La VTR se calcula como el peso de cada campo técnico sobre el total de patentes del país dividido entre el peso que ese mismo campo técnico tiene sobre el total de patentes del mundo. Cuando el valor de la VTR es superior a 1, se entiende que se trata de áreas técnicas donde hay especialización. Junto a la VTR se analizan también, para cada campo técnico, los pesos relativos de las patentes depositadas por cada agente sobre el total, ya que éstos expresan su aportación a la composición de la VTR (Urraca, 2005). La concentración de la distribución de competencias técnicas será medida a través del índice de Herfindahl-Hirschman (HHI).

La convergencia tecnológica puede ser medida a través de la Chi-cuadrado $\left(\chi^{2}\right)$ (Archibugi \& Pianta, 1992; Pianta \& Meliciani, 1996). Este indicador se define como

$$
\sum \frac{\left(\mathrm{p}_{\mathrm{j}}-\mathrm{pw}_{\mathrm{j}}\right)^{2}}{\mathrm{pw}_{\mathrm{j}}}
$$

donde $\mathrm{p}_{\mathrm{j}}$ es la cuota que representa el área técnica $\mathrm{j}$ en el total de patentes de un país y $\mathrm{pw}_{\mathrm{j}}$ la cuota que representa el área técnica $\mathrm{j}$ en el total de patentes 
del mundo. De acuerdo con su definición, la Chi-cuadrado mide la diferencia porcentual (o distancia) entre un país y el total de países de la base de datos en los pesos relativos que cada área técnica representa para el país y para el total. Cuanto menor la distancia, más se aproximará su valor a cero.

La Chi-cuadrado puede plantear dos problemas. En primer lugar, sobrevalora a los pequeños países que cuentan con una distribución de patentes muy estrecha. En este caso, el cuadrado de las diferencias puede ser substituido por el valor absoluto de las diferencias. Sin embargo, los resultados obtenidos difieren en muy escasa medida. En segundo lugar, el grado de especialización está relacionado negativamente con el tamaño de los países. Como el grado de especialización se calcula sobre la base de la distribución de las actividades tecnológicas en el mundo, cuanto mayor sea el país, más se verá afectada la distribución mundial de las patentes por área técnica, mayor será su similitud con la distribución mundial de patentes y menor será el índice de concentración. Para valorar este efecto, el cálculo de la desviación típica de las cuotas de patentes entre sectores para cada país respecto a la distribución de cuotas de patentes entre sectores para el total de la muestra ayuda a valorar el grado de robustez de la $\chi^{2}$. La correlación entre ambos indicadores fue 0.711 corroborándose la bondad de la $\chi^{2}$ como medida de convergencia tecnológica.

\section{Patrones de inserción de emn en países seguidores de Asia y América Latina.}

\subsection{Convergencia y Especialización tecnológica.}

La tabla 1 muestra algunos indicadores básicos que caracterizan el desempeño tecnológico y económico de estos países. Los indicadores tecnológicos muestran un mejor desempeño de los países asiáticos que los latinoamericanos, tanto en términos de resultados como de recursos. El indicador de número de patentes es especialmente bajo en los casos de China e India, considerando que su población pasaba del billón de habitantes en 2002, mientras que es especialmente elevada en Singapur donde, por el contrario, su población apenas superaba 4 millones de habitantes. en el mismo año.

Los países latinoamericanos muestran propensiones a patentar muy ba- 
TABLA 1

Indicadores tecnológicos y económicos del grupo de países analizado

\begin{tabular}{|c|c|c|c|c|c|c|c|}
\hline & \multicolumn{3}{|c|}{ Indicadores de tecnología } & \multicolumn{4}{|c|}{ Indicadores económicos } \\
\hline & Pat./1000hb & P\&D/PIB & Int.tech. & Apertura* & $\mathrm{PIBpc}$ & t.v.a & $\mathrm{PIBpc}^{* *}$ \\
\hline & 2000 & 2000 & $78-2003$ & 2000 & 2000 & 1985-95 & $1995-00$ \\
\hline Argentina & 0.012 & 0.44 & 44.42 & 22.22 & 11729.1 & 4.45 & 1.58 \\
\hline Brasil & 0.007 & 1.05 & 49.96 & 23.03 & 7744.7 & 3.47 & 1.50 \\
\hline Chile & 0.007 & 0.56 & 48.15 & 62.52 & 10388.9 & 9.18 & 1.82 \\
\hline Colombia & 0.002 & 0.18 & 62.65 & 42.33 & 5795.5 & 5.31 & 0.85 \\
\hline Cuba & 0.007 & 0,52 & 5.00 & - & - & -0.50 & - \\
\hline El Salvador & 0.010 & $0,08(\cdot)$ & 93.75 & 70.12 & 4622.4 & 5.31 & 1.10 \\
\hline Méjico & 0.005 & 0.37 & 63.35 & 64.69 & 9711.0 & 1.79 & 3.29 \\
\hline Venezuela & 0.006 & 0,37 & 61.15 & 46.30 & 7232.5 & 2.13 & 0.94 \\
\hline China & 0.001 & 1.00 & 48.36 & 48.36 & 3843.7 & 9.31 & 3.37 \\
\hline Corea del Sur & 0.161 & 2.39 & 10.98 & 87.18 & 14936.7 & 10.74 & 0.98 \\
\hline Hong-Kong & 0.103 & $0,25(\bullet)$ & 47.99 & 295.19 & 27892.5 & 7.79 & 1.21 \\
\hline India & 0.001 & $0,64(\bullet)$ & 55.56 & 30.45 & 2683.6 & 6.50 & 3.24 \\
\hline Singapur & 0.293 & 1.89 & 80.12 & 341.59 & 28643.6 & 9.23 & 2.08 \\
\hline Taiwán & 0.121 & 2.06 & 30.91 & - & - & 9.60 & - \\
\hline
\end{tabular}

(•): Datos para 1998.

(*): Cociente entre Exportaciones más Importaciones sobre PIB.

$\left({ }^{* *}\right)$ : Tasa de variación anual acumulativa del PIB per capita.

Fuente: Alan Heston, Robert Summers and Bettina Aten, Penn World Table Version 6.1, Center for International Comparisons at the University of Pennsylvania (CICUP), October 2002; "Main Science and Technology indicators", OCDE; "Espace Bulletin 1978-02" y actualización para 2003, European Patent Office; Indicadores de la red RICyT http://www.ricyt.oy; site http://www.science.oy.hk. El dato de población para calcular el ratio patentes por cada 1000 habitantes para Taiwán fue extraído del site "wikipedia", julio de 2006 y para Cuba se utilizaron las estimaciones para 2003 o 2004 de las Naciones Unidas. 
jas, en términos relativos, sin llegar en ningún caso a 0,1 patentes por 1000 HB. Con relación al esfuerzo tecnológico, se comprueba también un mejor desempeño de los países asiáticos. Salvo en los casos de Hong-Kong e India, los gastos en I+D superan al 1 por ciento del PIB en el conjunto asiático (con media 1,37), siendo especialmente elevados los valores registrados por Corea del Sur y Taiwán, mientras que en el conjunto latinoamericano (con media 0,44), apenas Brasil supera el simbólico 1 por ciento.

El grado de internacionalización tecnológica ${ }^{3}$ es mayor en los países latinoamericanos que en los asiáticos. En media, en los países latinoamericanos (exceptuando Cuba), seis de cada diez patentes son internacionalizadas en cuanto que en Asia, son 4,5 de cada diez. Este encuentro resulta especialmente revelador cuando se compara con los datos de apertura económica, ya que, en los países asiáticos, el grado de apertura es, en media, 1,6 veces el PIB, en cuanto que en los países latinoamericanos es 0,47 veces el valor del PIB. Esto significa que la apertura económica no necesariamente implica internacionalización tecnológica. Países con elevados índices de apertura pueden detentar competencias tecnológicas en los agentes locales en cuanto que países menos abiertos, pueden tener sus competencias tecnológicas en manos de agentes extranjeros.

Finalmente, los indicadores de desempeño económico también muestran mejores resultados para los países asiáticos que para los países latinoamericanos, los cuales registran niveles de PIBpc (Producto Interno Bruto per capita) apenas superiores a los de China e India para el año 2000. En media (exceptuando Cuba y Taiwán para los cuales no se cuenta con información), el PIBpc de los países latinoamericanos es de 8.175 dólares, en cuanto que para los países asiáticos es de 15.600 dólares, casi el doble. Esta observación se debe, principalmente, a los elevados niveles de desarrollo alcanzados para el año 2000 por Corea del Sur, Hong-Kong y Taiwán, cuyos ritmos de crecimiento fueron muy elevados durante la década 85-95 (notablemente superiores a los registrados por los países latinoamericanos con la excepción de Chile). Durante la última mitad de los años noventa, los ritmos de crecimiento

\footnotetext{
3 Medida como el número de patentes "internacionalizadas" sobre el total. Se considera que una patente es internacionalizada cuando al menos un depositante o al menos un inventor tiene su residencia en un país diferente al de la nacionalidad de la patente.
} 
TABLA 2

Convergencia tecnológica (valores del la $\chi^{2}$ )

\begin{tabular}{|c|c|c|c|c|c|c|}
\hline \multicolumn{5}{|c|}{ Países seguidores } & \multicolumn{2}{|c|}{ Países líderes } \\
\hline & $1978-1985$ & 1986-1995 & $1996-2003$ & $1978-2003$ & & $1978-2003$ \\
\hline \multicolumn{5}{|c|}{ AMÉRICA LATINA } & Estados Unidos & 8,4 \\
\hline Argentina & 128,6 & 64,1 & 94,4 & 79,1 & Japón & 15,4 \\
\hline Brasil & 69,4 & 59,6 & 61,2 & 44,0 & Alemania & 7,6 \\
\hline Chile & 1107,5 & 235,3 & 147,7 & 114,7 & Francia & 5,9 \\
\hline Colombia & 135,0 & 410,8 & 220,5 & 131,7 & Gran Bretaña & 2,8 \\
\hline Cuba & - & 774,2 & 333,1 & 489,0 & Bélgica & 18,0 \\
\hline El Salvador & 501,2 & 96,8 & 68,9 & 55,4 & Italia & 30,7 \\
\hline Méjico & 187,6 & 110,7 & 34,1 & 39,5 & Holanda & 14,2 \\
\hline Venezuela & 842,3 & 191,8 & 853,5 & 436,2 & & \\
\hline
\end{tabular}

A S I A

\begin{tabular}{|c|c|c|c|c|}
\hline China & 161,7 & 16,1 & 20,9 & 16,2 \\
\hline Corea del Sur & 84,4 & 46,0 & 30,8 & 42,0 \\
\hline Hong-Kong & 93,8 & 155,1 & 129,5 & 133,0 \\
\hline India & 103,6 & 127,4 & 90,2 & 89,2 \\
\hline Singapur & 326,1 & 63,9 & 53,2 & 54,9 \\
\hline Taiwán & 68,6 & 55,3 & 126,2 & 56,7 \\
\hline \multicolumn{5}{|l|}{ U ROPA } \\
\hline Portugal & - & - & - & 33,8 \\
\hline España & - & - & - & 28,7 \\
\hline
\end{tabular}

Fuente: "Espace Bulletin 1978-02" y actualización para 2003, EPO y elaboración propia. 
se desaceleraron para prácticamente todos los países con las excepciones de India, China y Méjico.

El crecimiento económico y tecnológico de los países examinados durante el periodo 1978-2000 fue acompañado de un aumento de la convergencia tecnológica con los países líderes (Tabla 2). Prácticamente, todos los países redujeron sus distancias con el mundo, excepto Venezuela, Taiwán y HongKong. El país que, para todo el periodo, presenta una distancia menor es China (16.2), lo cual es esperado dado el tamaño de su economía. Economías grandes son más diversificadas económicamente y, por ende, tecnológicamente. Le sigue, con distancias entre 40 y 56 puntos, un primer grupo formado por Méjico, Brasil, El Salvador, Corea del Sur, Singapur y Taiwán. Se trata de países que todavía no han completado su proceso de captura mostrando mayores distancias con respecto a la distribución de competencias del mundo.

Un segundo grupo de países, con distancias de 79,1 y 89,2 puntos, incluye a Argentina e India, respectivamente. En este caso, el efecto del retraso tecnológico sobre la especialización es mayor, lo que lleva a unas distancias también mayores. Finalmente, un tercer grupo, con distancias superiores a 100 puntos incluye a Chile, Colombia, Cuba, Venezuela y Hong-Kong. Se trata de países fuertemente especializados que, habiendo o no reducido su desfase con los países líderes, muestran una importante asimetría tecnológica en un amplio número de campos técnicos con respecto al mundo. La gravedad de esta asimetría dependerá de en dónde estos países se especializan. Si los países se especializan en campos técnicos poco dinámicos y permeables para el desarrollo de otras tecnologías, el proceso de captura estará comprometido en el futuro, sometiéndose a un más que probable letargo tecnológico. Si por el contrario, los países se especializan en campos técnicos con elevados grados de oportunidad tecnológica y de permeabilidad con otras tecnologías, las fortalezas desarrolladas en esos campos técnicos permitirán el desarrollo de otros, acelerándose el proceso de captura y de convergencia.

Para evaluar este efecto se elaboró la Tabla 3, donde se presentan los campos técnicos en los que los países registran VTR superiores a 1,5. Junto a éstos, son identificados los campos técnicos en donde las distancias mantenidas con los países líderes son mayores por sobre-especialización con una variación porcentual superior al 100 del peso registrado por el mundo (lo que 
TABLA $3 a$

Análisis de la especialización nacional - America Latina

\begin{tabular}{|c|c|c|c|}
\hline & Sectores VTR > 1,5 & Sobre-especialización > 100\% & Subespecialización > 60\% \\
\hline Argentina & $\begin{array}{l}\text { Agricultura, Alimentos y } \\
\text { Tabaco, Artículos de uso } \\
\text { personal y juguetes, Artículos } \\
\text { de la Salud y Salvavidas, } \\
\text { Minas, Motores y Bombas, } \\
\text { Armas }\end{array}$ & $\begin{array}{l}\text { Agricultura, Alimentos y } \\
\text { Tabaco, Artículos de uso } \\
\text { personal y juguetes, } \\
\text { Artículos de la Salud } \\
\text { y Salvavidas }\end{array}$ & $\begin{array}{l}\text { Impresión, Química del } \\
\text { petróleo y carbono, } \\
\text { Ingeniería y Física Nuclear, } \\
\text { Electricidad, Electrónica }\end{array}$ \\
\hline Brasil & $\begin{array}{l}\text { Alimentos y Tabaco, Artículos } \\
\text { de salud y salvavidas, Química } \\
\text { del petróleo y carbono, } \\
\text { Metalurgia, Papel y Celulosa, } \\
\text { Minas, Motores y Bombas, } \\
\text { Ingeniería }\end{array}$ & $\begin{array}{l}\text { Papel y Celulosa, Minas, } \\
\text { Motores y Bombas }\end{array}$ & $\begin{array}{l}\text { Armas, Ingeniería y Física } \\
\text { Nuclear, Electrónica }\end{array}$ \\
\hline Chile & $\begin{array}{l}\text { Agricultura, Alimentos y } \\
\text { Tabaco, Artículos de uso } \\
\text { personal y juguetes, } \\
\text { Separación y Mezclas, } \\
\text { Bioquímica, Metalurgia, } \\
\text { Textil e mat. Flexibles, Papel } \\
\text { y Celulosa, Motores y } \\
\text { Bombas, Armas }\end{array}$ & $\begin{array}{l}\text { Agricultura, Alimentos } \\
\text { y Tabaco, Bioquímica, } \\
\text { Metalurgia, Motores } \\
\text { y Bombas, Armas }\end{array}$ & $\begin{array}{l}\text { Impresión, Transporte, } \\
\text { Química del petróleo } \\
\text { y carbono, Minas, } \\
\text { Ingeniería y Física Nuclear, } \\
\text { Electricidad, Electrónica. }\end{array}$ \\
\hline Colombia & $\begin{array}{l}\text { Agricultura, Artículos de salud } \\
\text { y salvavidas, Química orgánica } \\
\text { e inorgánica, Química del } \\
\text { petróleo y carbono, } \\
\text { Bioquímica, Textil e mat. } \\
\text { Flexibles, Papel y Celulosa }\end{array}$ & $\begin{array}{l}\text { Agricultura, Artículos } \\
\text { de salud y salvavidas, } \\
\text { Papel y Celulosa }\end{array}$ & $\begin{array}{l}\text { Alimentos y Tabaco, } \\
\text { Transporte, Minas, } \\
\text { Ingenieria, Iluminación } \\
\text { y Refrigeración, Armas, } \\
\text { Instrumentos, Ingeniería y } \\
\text { Física Nuclear, Electrónica }\end{array}$ \\
\hline Cuba & $\begin{array}{l}\text { Agricultura, Artículos de } \\
\text { la Salud y Salvavidas, } \\
\text { Bioquímica }\end{array}$ & $\begin{array}{l}\text { Agricultura, Artículos } \\
\text { de la Salud y Salvavidas, } \\
\text { Bioquímica }\end{array}$ & $\begin{array}{l}\text { Todos los sectores, } \\
\text { (exceptuando Química } \\
\text { orgánica e inorgánica, } \\
\text { Metalurgia e Instrumentos). }\end{array}$ \\
\hline Méjico & $\begin{array}{l}\text { Alimentos y Tabaco, Artículos } \\
\text { de la Salud y Salvavidas, } \\
\text { Bioquímica, Metalurgia, Papel } \\
\text { y Celulosa }\end{array}$ & $\begin{array}{l}\text { Alimentos y Tabaco, } \\
\text { Bioquímica, Metalurgia, } \\
\text { Papel y Celulosa }\end{array}$ & $\begin{array}{l}\text { Armas, Instrumentos, } \\
\text { Ingeniería y Física Nuclear }\end{array}$ \\
\hline El Salvador & $\begin{array}{l}\text { Agricultura, Alimentos y } \\
\text { Tabaco, Artículos de la Salud y } \\
\text { Salvavidas, Máquinas y } \\
\text { Herramientas, Construcción, } \\
\text { Electrónica }\end{array}$ & $\begin{array}{l}\text { Agricultura, Alimentos } \\
\text { y Tabaco, Máquinas y } \\
\text { Herramientas }\end{array}$ & $\begin{array}{l}\text { Impresión, Química del } \\
\text { petróleo y carbono, Textil } \\
\text { e mat. Flexibles, Papel y } \\
\text { Celulosa, Minas, Iluminación } \\
\text { y Refrigeración, Armas, } \\
\text { Ingeniería y Física Nuclear }\end{array}$ \\
\hline Venezuela & $\begin{array}{l}\text { Artículos de la Salud y } \\
\text { Salvavidas, Separación y } \\
\text { Mezclas, Química del petróleo } \\
\text { y carbono, Bioquímica, } \\
\text { Metalurgia, Minas }\end{array}$ & $\begin{array}{l}\text { Química del petróleo } \\
\text { y carbono, Metalurgia, } \\
\text { Minas }\end{array}$ & $\begin{array}{l}\text { Impresión, Transporte, } \\
\text { Papel y Celulosa, Armas, } \\
\text { Ingeniería y Física Nuclear, } \\
\text { Electricidad, Electrónica }\end{array}$ \\
\hline
\end{tabular}

Fuente: "Espace Bulletin 1978-02" y actualización para 2003, EPO y elaboración propia. 
Patrones de Inserción de las Empresas Multinacionales en la

Formación de Competencias Tecnológicas de Países Seguidores

TABLA 3b

Análisis de la especialización nacional - Asia

Sectores VTR $>1,5$

China

Corea del Sur

Electrónica

Artículos de uso personal y juguetes, Separación y Mezclas, Química del petróleo y carbono, Bioquímica

Hong-kong Artículos de uso personal y juguetes, Impresión, Electricidad

India

Singapur

Alimentos y Tabaco, Bioquímica, Armas, Electricidad, Electrónica

Artículos de salud y salvavidas, Química orgánica e inorgánica, Química del petróleo y carbono, Bioquímica salvavidas, Química orgánica
Artículos de salud y e inorgánica, Bioquímica

Artículos de uso personal y juguetes, Impresión

Artículos de uso personal y juguetes, Química del petróleo y carbono

Electrónica

Alimentos y Tabaco, Minas Armas, Electricidad, Electrónica
Minas, Armas

Separación y Mezclas, Máquinas y Herramientas, Química del petróleo y del carbono, Papel y Celulosa, Minas, Ingeniería y Física Nuclear

Química orgánica e inorgánica, Bioquímica, Metalurgia, Papel y Celulosa, Ingenieria, Armas, Ingeniería y Física Nuclear

Artículos de uso persona y juguetes, Máquinas y Herramientas, Impresión, Transporte, Constriucción, Ingeniería, Iluminación y Refrigeración, Armas, Ingeniería y Física Nuclear

Transporte, Motores y Bombas, Ingeniería, lluminación y refrigeración, Ingeniería y Física Nuclear

Química orgánica e inorgánica, Metalurgia, Papel y Celulosa, Minas juguetes, Construcción,

Artículos de uso personal y juguetes
Iluminación y Refrigeración, Electricidad

Fuente: "Espace Bulletin 1978-02" y actualización para 2003, EPO y elaboración propia. 
significa que el peso en el país seguidor es, como mínimo, el doble del registrado en el mundo); o por sub-especialización, con una variación porcentual superior al 60 por ciento (lo que significa que el peso del país seguidor, en ese campo técnico, es inferior al 40 por ciento del que registra el mundo).

Los resultados son reveladores de la existencia de un patrón de especialización nacional bien diferenciado entre Asia y América Latina. Algunas observaciones interesantes son:

(i) Con carácter general, los países latinoamericanos se especializan en campos técnicos relacionados con una estructura productiva intensiva en recursos naturales y en factor trabajo (Agricultura, Alimentos y Tabaco, Artículos de la Salud y Salvavidas, Química del Petróleo y del Carbono, Minas, Metalurgia o Papel y Celulosa), siendo Bioquímica el único campo técnico intensivo en conocimiento registrado en cinco de los seis países latinoamericanos analizados. Algunos campos técnicos, relacionados o no con este patrón, atienden a particularidades relativas a cada país. Son los casos de Textil y Materiales Flexibles, en Chile y Colombia; Motores y Bombas en Argentina, Brasil y Chile; o, Ingeniería en Brasil. Mientras, los países asiáticos siguen una especialización tecnológica asociada a una estructura productiva intensiva en factor trabajo y en conocimiento (Artículos de uso personal y Juguetes, Bioquímica, Electricidad y Electrónica). Algunas especificidades nacionales elevan la importancia de ciertos campos técnicos, como son los casos de Química del Petróleo y del Carbono en China e India, Impresión en Hong-Kong; Alimentos y Tabaco en Singapur o Iluminación y Refrigeración en Taiwán;

(ii) Los países latinoamericanos se especializan en un mayor número de campos técnicos que los países asiáticos. Sin embargo, el número de campos técnicos que registran sobre-especialización se reduce bastante en los países latinoamericanos mientras que se mantiene, en buena parte, en los países asiáticos. Este hecho revela que las sobre-especializaciones en América Latina son mucho más marcadas que en Asia;

(iii) El análisis de la sub-especialización revela que los campos técnicos donde los países latinoamericanos mantienen mayores distancias con el mundo son precisamente los vinculados a sectores de mayor dinamismo y los que, por contar con un elevado grado de permeabilidad, impulsan el 
desarrollo de otras tecnologías. Son los casos de las Ingenierías, Instrumentos, Electricidad y Electrónica. Los ejemplos de Chile, Colombia, Cuba y El Salvador son especialmente graves en la medida en que son numerosos los sectores donde existe escasa o nula actividad tecnológica. Los países asiáticos, por su parte, registran un escaso dinamismo tecnológico en un mayor número de campos técnicos, pero estos están más vinculados a sectores intensivos en recursos naturales (Minas, Papel y Celulosa y, en cierto sentido, Metalurgia) o de baja permeabilidad (Armas y Municiones o Transporte), siendo destacables las lagunas observadas en campos técnicos relevantes como son Máquinas y Herramientas, algunas tecnologías Químicas y las Ingenierías.

\subsection{EMN y formación de competencias en países seguidores.}

Con el objetivo de identificar el papel de las EMN en la formación de competencias tecnológicas nacionales, se estimo la distribución de la cuota de patentes de acuerdo con la residencia del depositante, distinguiendo entre Empresas Nacionales (EN), ${ }^{4}$ Empresas Multinacionales (EMN), Empresas sin clasificar (SC); Investigadores Independientes (II), Agentes Públicos (CPI), que incluye Universidades, Centros Públicos de Investigación, Fundaciones o Agencias Públicas residentes y no residentes y Cooperaciones (COOPS) o patentes depositadas por más de un depositante.

Los resultados presentados en la Tabla 4 muestran la intensidad de la inserción de las EMN como usuarios de los recursos tecnológicos localizados en cada país. Dicha intensidad es medida como la cuota de patentes depositadas por EMN en cuya producción participó al menos un inventor con residencia en ese determinado país. De acuerdo con este criterio, se identifica un primer grupo formado por Cuba y Corea del Sur, donde las EMN depositan menos del $10 \%$ de las patentes cuyos inventores residen en estos países. A pesar de que en ambos países las EMN tienen un escaso papel en la formación de competencias, existe una gran diferencia entre ambos. En Corea del Sur, las competencias nacionales se localizan en empresas nacionales (65,5\%), en cuanto que en Cuba

4 En el recuento de Hong-Kong, las firmas residentes en China o subsidiarias de firmas chinas residentes en HongKong fueron consideradas como nacionales y en el recuento de China, las firmas residentes en Hong-Kong o sus subsidiárias fueron consideradas nacionales. 
TABLA 4

Número de patentes y distribución de pesos por agente depositante: concentración de la distribución de pesos por campo técnico y distancia entre EMN y país

\begin{tabular}{|c|c|c|c|c|c|c|c|c|c|c|c|}
\hline & COOPS & $\mathrm{CPI}$ & CPIEXT & EMN & EN & II & SC & $\begin{array}{l}\text { Total } \\
1978- \\
2003\end{array}$ & $\begin{array}{l}\mathrm{HHI} \\
\text { EMN }\end{array}$ & $\begin{array}{l}\text { HHI } \\
\text { País }\end{array}$ & $\begin{array}{c}\text { Distancia } \\
\text { EMN } \\
\left(\chi^{2}\right)\end{array}$ \\
\hline & [1] & [2] & [3] & [4] & [5] & [6] & [7] & [8] & [9] & {$[10]$} & [ 111$]$ \\
\hline Argentina & 2.9 & 1,1 & 4,7 & 32,8 & 8,3 & 45,5 & 4,7 & 448 & 0,14 & 0,12 & 48,6 \\
\hline Brasil & 2.8 & 3,1 & 2,0 & 44,0 & 26,8 & 18,9 & 2,4 & 1229 & 0,10 & 0,07 & 304,4 \\
\hline Chile & 3.7 & 0,0 & 8,3 & 32,4 & 10,2 & 38,9 & 6,5 & 108 & 0,16 & 0,08 & 221,3 \\
\hline Colombia & 2.4 & 2,4 & 3,6 & 48,2 & 7,2 & 36,1 & 0,0 & 83 & 0,20 & 0,14 & 308,1 \\
\hline Cuba & 2.5 & 90,0 & 0,0 & 1,3 & 5,0 & 1,3 & 0,0 & 80 & 1,00 & 0,25 & 206,4 \\
\hline Méjico & 4.9 & 2,1 & 5,1 & 51,7 & 13,8 & 20,8 & 1,7 & 472 & 0,12 & 0,09 & 157,4 \\
\hline El Salvador & 1.6 & 0,0 & 0,0 & 92,2 & 0,0 & 6,3 & 0,0 & 64 & 0,10 & 0,09 & 116,6 \\
\hline Venezuela & 7.0 & 0,0 & 5,7 & 43,3 & 28,7 & 15,3 & 0,0 & 157 & 0,10 & 0,09 & 184,3 \\
\hline Corea del Sur & 3.5 & 3,9 & 0,1 & 7,9 & 65,5 & 11,8 & 7,4 & 7593 & 0,13 & 0,14 & 61,5 \\
\hline China & 8.9 & 8,4 & 3,9 & 36,9 & 8,7 & 22,6 & 10,7 & 1733 & 0,11 & 0,09 & 99,0 \\
\hline Hong-Kong & 2.6 & 1,3 & 0,4 & 40,4 & 29,7 & 22,8 & 2,9 & 698 & 0,12 & 0,13 & 443,1 \\
\hline India & 4.7 & 14,6 & 1,6 & 48,3 & 24,0 & 6,0 & 0,7 & 1224 & 0,19 & 0,22 & 476,0 \\
\hline Singapur & 3.4 & 8,1 & 1,4 & 72,8 & 7,2 & 5,4 & 1,7 & 1177 & 0,16 & 0,13 & 476,0 \\
\hline Taiwán & 1.5 & 2,0 & 1,1 & 24,9 & 15,0 & 52,5 & 2,9 & 2776 & 0,11 & 0,09 & 72,3 \\
\hline
\end{tabular}

Fuente: "Espace Bulletin 1978-02" y actualización para 2003, EPO y elaboración propia.

se concentran fuertemente en agentes públicos (90,0\%). Este hecho tiene una implicación. En la medida que el sistema productivo es más diversificado tecnológicamente que el sector público, la distribución de las cuotas por campo técnico deberán ser más concentradas en Cuba, lo que explica su mayor índice de HHI respecto del resto de países (columna 10 de la Tabla 4).

Un segundo grupo de países, incluyendo a Argentina, Chile y Taiwán, se caracteriza por una cuota de patentes depositadas por EMN más elevada (entre un $24 \%$ y un $32 \%$ ) y por un liderazgo de la concentración de las com- 
petencias en los investigadores independientes (hasta un 50\%). Este patrón revela un Sistema Nacional de Innovación desarticulado, donde los esfuerzos innovadores no se encuentran vinculados a instituciones y donde las relaciones entre agentes son muy débiles.

Un tercer grupo estaría formado por países donde las EMN lideran los procesos de innovación. Con cuotas que varían entre un $40 \%$ y un $50 \%$ se encuentran Brasil, Colombia, Méjico, Hong-Kong e India y con cuotas muy elevadas se encuentran Singapur (72,8\%) y El Salvador (92,2\%). En estos últimos países, prácticamente se puede concluir que quienes explotan los recursos tecnológicos nacionales son las EMN.

Las columnas 9 y 10 de la tabla 4 presentan la concentración de la distribución de la cuota de patentes por campo técnico depositadas por EMN y para el total nacional, respectivamente. En principio, dado que la muestra de EMN en cada país no es representativa de ninguna base productiva y que la internacionalización de la I+D está fundamentada, en alguna medida, en la explotación de ventajas locales, las EMN deberán tener una distribución de cuotas por campo técnico más concentrada, operando de forma más intensiva en un menor número de campos técnicos. Este resultado es el observado para la mayor parte de los países analizados excepto en tres - Corea del Sur, HongKong e India -, donde los países son, de por sí, altamente especializados en un número reducido de campos técnicos.

La última columna de la Tabla 4 presenta el indicador $\chi^{2}$ midiendo la distancia entre la distribución de cuotas de patentes depositadas por EMN y la registrada para el resto de agentes nacionales. Cuanto más próximo se encuentre el indicador de cero, más enfocada estará la internacionalización a la explotación de ventajas nacionales y cuanto más distante, más enfocada estará a la explotación de ventajas adquiridas en casa. La $\chi^{2}$ muestra en todos los casos valores elevados, lo cual es un indicio de que las EMN utilizan recursos tecnológicos de países seguidores de acuerdo con las ventajas adquiridas en casa y no con las ventajas desarrolladas por el país.

Para analizar este indicio con un mayor grado de detalle, se elaboró la Tabla 5. En ella, aparecen los campos técnicos caracterizados como Competencias Centrales (C), Competencias Nicho (N), Competencias de Fondo (o "background”) (B) y Competencias Marginales (M) (Patel \& Pavitt, 1997). Las "Competencias Centrales" vienen representadas por aquellos campos técnicos 
en donde el país registró VTR superior a 1,5 y un peso superior a $4,2 \% .^{5}$ Las competencias centrales componen las fortalezas tecnológicas del país.

Los campos técnicos en donde el país está especializado $(\mathrm{VTR}>1,5)$ pero cuya representatividad es pequeña (detenta un peso inferior a 4,2) componen las "Competencias Nicho". Las "Competencias de Fondo" y las "Competencias Marginales" son caracterizadas por los campos técnicos en donde no existe especialización $(\mathrm{VTR}<1,5)$. Si el peso representado por el campo técnico fuese mayor a 4,2, él constituirá una Competencia de Fondo. Si fuese menor a 4,2, se tratará de una Competencia Marginal.

Una vez caracterizados los diferentes tipos de competencias, fue calculada la contribución de las EMN (peso) en cada campo técnico. Se consideró que una competencia nacional habría sido construida por EMN cuando el peso se mostraba superior al 60 por ciento. Son los casos destacados en negrita. Si las EMN forman competencias centrales o nicho, ellas están contribuyendo a configurar la especialización tecnológica del país. Si forman competencias de fondo o marginales, estarán contribuyendo para la diversificación tecnológica del país. Como es de esperar, la contribución de las EMN en la formación de competencias nacionales depende de la intensidad de su inserción en el sistema de innovación.

De acuerdo con estos criterios, el patrón de inserción de EMN de acuerdo con su contribución a la formación de competencias es:

- Grupo 1, formado por Cuba, China, Corea del Sur y Taiwán. En este grupo, las EMN no contribuyen significativamente en ningún campo técnico a la formación de competencias nacionales;

- Grupo 2, formado por El Salvador, Singapur, Chile, Colombia y Venezuela. En los casos de El Salvador y Singapur, la formación de competencias nacionales está prácticamente en manos de las EMN, responsables por la formación de todo tipo de competencias, tanto las que contribuyen a la especialización como las que contribuyen a la diversificación. Chile, Colombia y Venezuela responden al mismo patrón a pesar de que las EMN representen un peso menor.

- Grupo 3, formado por India y Hong-Kong. Este grupo se caracteriza por

5 El valor 4,2 es el peso medio que tendría cada sector si hubiera una perfecta distribución del 100\% del total de patentes entre los 24 campos técnicos contemplados. 
un papel activo de las EMN en la formación de competencias de fondo y marginales, contribuyendo para la diversificación tecnológica nacional. La diversificación camina hacia las tecnologías químicas en HongKong y a tecnologías de Impresión, Ingeniería nuclear o Electricidad en la India. En este último país, las EMN componen también competencias de fondo en tecnologías estratégicas, como son Instrumentos y Electrónica. El único caso observado de contribución a la especialización es en HongKong, donde las EMN forman una competencia central en Impresión. - Grupo 4, formado por Argentina y Brasil. En estos países, las EMN contribuyen para la especialización tecnológica formando competencias nicho. Son los casos de Minas, Armas y Municiones en Argentina y Alimentos y Tabaco, Metalurgia y Papel y Celulosa en Brasil.

Un último análisis permite identificar patrones de inserción desde el punto de vista del aprovechamiento de las competencias tecnológicas de los países receptores. Para ello, será comparada la distribución de los tipos de competencias nacionales con la especialización tecnológica de las EMN que utilizan recursos de estos países (tabla 5). Se constata aprovechamiento de fortalezas nacionales si las EMN se especializan con respecto al mundo $(\mathrm{VTR}>1,5)$ en tecnologías donde el país también está especializado (competencias centrales y competencias nicho).

De acuerdo con estos criterios, se observan tres patrones. Uno, formado por Corea del Sur y Cuba, donde no existe aprovechamiento de las ventajas locales (Corea del Sur) o lo es en muy escasa medida (Cuba sólo en Bioquímica). El segundo, formado por Argentina, Brasil, Méjico e India, donde las EMN se especializan en prácticamente todas sus competencias centrales y nicho, lo cual indica aprovechamiento de las ventajas que el país ofrece. Apenas dos excepciones. El desaprovechamiento de Motores y Bombas para Brasil y de Metalurgia en Méjico.

El tercer patrón, formado por el resto de los países, recoge un balance más compensado entre las competencias centrales y nicho que son aprovechadas y que no lo son. En términos generales, las EMN aprovechan más las competencias centrales que las competencias nicho, siendo casi siempre mayor el número de competencias explotadas donde hay especialización que el de las no explotadas. 
TABLA 5

Contribución y aprovechamiento de ventajas locales de acuerdo con el tipo de ventaja

\begin{tabular}{|c|c|c|c|c|c|c|c|c|c|c|c|c|c|}
\hline & & $\begin{array}{l}\frac{\pi}{3} \\
\frac{5}{3} \\
\frac{3}{5} \\
\frac{5}{4}\end{array}$ & 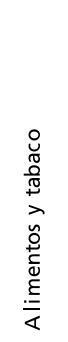 & 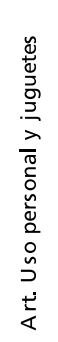 & 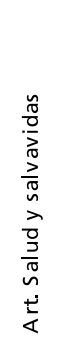 & 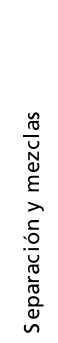 & 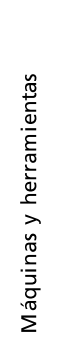 & $\begin{array}{l}\frac{c}{0} \\
\frac{0}{a} \\
\frac{a}{\varrho} \\
\underline{E}\end{array}$ & 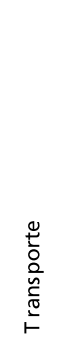 & 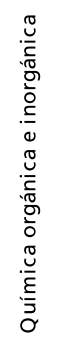 & 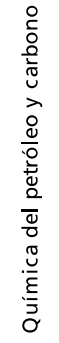 & $\begin{array}{l}\frac{0}{\tilde{E}} \\
\frac{\sigma}{5} \\
\frac{.0}{\infty}\end{array}$ & 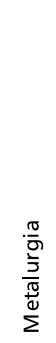 \\
\hline & VTR-EMN & 2.73 & 2.34 & 2.27 & 3.68 & 0.63 & 0.25 & 0.00 & 1.01 & 1.10 & 0.00 & 1.76 & 0.38 \\
\hline \multirow{2}{*}{ ARGENTINA } & CLASE & $\mathrm{N}$ & $\mathrm{N}$ & C & C & M & $\mathrm{M}$ & $M$ & B & B & $\mathrm{M}$ & $\mathrm{M}$ & $M$ \\
\hline & VTR-EMN & 1.85 & 2.70 & 0.68 & 1.68 & 0.96 & 0.71 & 0.10 & 0.73 & 1.65 & 2.28 & 0.34 & 2.48 \\
\hline \multirow[t]{2}{*}{ BRASIL } & CLASE & M & $\mathbf{N}$ & $M$ & C & M & B & M & B & B & $\mathrm{N}$ & M & $\mathbf{N}$ \\
\hline & VTR-EMN & 5.72 & 0.00 & 1.06 & 1.08 & 3.50 & 0.52 & 0.00 & 0.00 & 2.11 & 0.00 & 3.16 & 1.59 \\
\hline \multirow[t]{2}{*}{ CHILE } & CLASE & c & $\mathrm{N}$ & c & B & C & B & M & M & B & $\mathrm{M}$ & C & $C$ \\
\hline & VTR-EMN & 8.35 & 0.00 & 0.00 & 1.26 & 1.53 & 0.00 & 1.31 & 0.31 & 2.69 & 0.00 & 1.85 & 1.40 \\
\hline \multirow[t]{2}{*}{ COLOMBIA } & CLASE & C & $M$ & $M$ & C & M & $M$ & M & $\mathrm{M}$ & C & $\mathrm{N}$ & C & M \\
\hline & VTR-EMN & 0.00 & 0.00 & 0.00 & 0.00 & 0.00 & 0.00 & 0.00 & 0.00 & 0.00 & 0.00 & 36.91 & 0.00 \\
\hline \multirow[t]{2}{*}{ CUBA } & CLASE & c & $M$ & M & c & M & M & M & M & B & $M$ & C & $M$ \\
\hline & VTR-EMN & 1.37 & 2.11 & 0.91 & 1.86 & 0.38 & 0.82 & 0.65 & 1.27 & 1.73 & 0.00 & 1.97 & 1.14 \\
\hline \multirow[t]{2}{*}{ MEXICO } & CLASE & M & $\mathrm{N}$ & M & C & M & M & M & B & B & $\mathrm{M}$ & C & $\mathrm{C}$ \\
\hline & VTR-EMN & 1.13 & 4.37 & 1.26 & 1.49 & 1.04 & 2.79 & 0.00 & 1.26 & 0.34 & 0.00 & 0.63 & 0.95 \\
\hline \multirow[t]{2}{*}{ SALVADOR } & CLASE & $\mathrm{N}$ & C & M & C & M & C & M & B & B & M & $\mathbf{M}$ & M \\
\hline & VTR-EMN & 0.00 & 0.00 & 0.00 & 2.22 & 1.35 & 2.15 & 0.77 & 0.55 & 1.09 & 2.27 & 0.00 & 4.92 \\
\hline \multirow[t]{2}{*}{ VENEZUELA } & CLASE & M & M & M & c & C & B & M & M & B & C & C & C \\
\hline & VTR-EMN & 0.42 & 1.48 & 1.62 & 1.01 & 1.68 & 0.69 & 0.82 & 0.41 & 1.24 & 1.93 & 1.27 & 0.52 \\
\hline \multirow[t]{2}{*}{ CHINA } & CLASE & M & $M$ & C & B & C & B & M & M & B & $N$ & C & M \\
\hline & VTR-EMN & 0.89 & 0.58 & 0.99 & 0.91 & 0.21 & 0.67 & 0.35 & 0.40 & 1.70 & 0.00 & 2.60 & 1.50 \\
\hline \multirow[t]{2}{*}{ COREA SUR } & CLASE & M & M & M & B & M & M & M & B & B & M & M & M \\
\hline & VTR-EMN & 1.18 & 0.91 & 5.65 & 0.36 & 0.22 & 0.39 & 3.54 & 0.66 & 0.43 & 1.09 & 0.52 & 0.00 \\
\hline \multirow[t]{2}{*}{ HONG-KONG } & CLASE & M & M & C & B & M & $\mathrm{M}$ & C & B & $\mathbf{M}$ & $\mathbf{M}$ & M & $\mathrm{M}$ \\
\hline & VTR-EMN & 0.56 & 0.87 & 0.13 & 1.62 & 0.47 & 0.31 & 0.18 & 0.15 & 2.42 & 0.78 & 2.31 & 0.57 \\
\hline \multirow[t]{2}{*}{ INDIA } & CLASE & M & M & M & c & M & M & $\mathbf{M}$ & M & c & $\mathrm{N}$ & c & M \\
\hline & VTR-EMN & 0.55 & 2.81 & 0.35 & 0.40 & 0.50 & 0.55 & 1.53 & 0.32 & 0.56 & 1.08 & 0.52 & 0.98 \\
\hline \multirow[t]{2}{*}{ SINGAPUR } & CLASE & M & $\mathbf{N}$ & $M$ & B & M & $\mathbf{M}$ & $\mathbf{M}$ & $\mathbf{M}$ & B & $\mathbf{M}$ & c & M \\
\hline & VTR-EMN & 0.58 & 0.37 & 2.47 & 0.82 & 0.62 & 0.90 & 0.46 & 0.81 & 0.74 & 0.89 & 0.48 & 0.73 \\
\hline TAIWÁN & CLASE & M & M & C & B & M & B & M & B & B & M & M & M \\
\hline
\end{tabular}


TABLA 5

Contribución y aprovechamiento de ventajas locales de acuerdo con el tipo de ventaja (cont.)

\begin{tabular}{|c|c|c|c|c|c|c|c|c|c|c|c|c|c|}
\hline & & 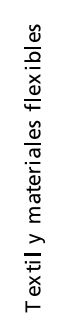 & 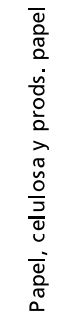 & 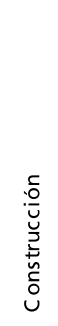 & $\stackrel{\stackrel{n}{\mathscr{J}}}{\stackrel{.}{\Sigma}}$ & 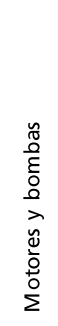 & $\begin{array}{l}\frac{\pi}{\frac{\sigma}{\sigma}} \\
\frac{\sigma}{\sigma} \\
\frac{\sigma}{\Xi} \\
\stackrel{\sigma}{\Xi}\end{array}$ & 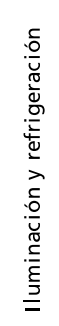 & 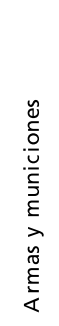 & 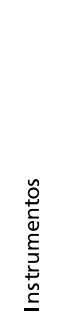 & 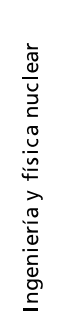 & 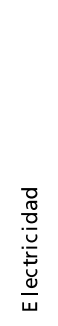 & 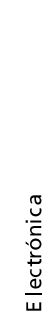 \\
\hline & VTR-EMN & 0.45 & 2.73 & 0.00 & 4.19 & 1.03 & 0.40 & 0.98 & 3.34 & 0.48 & 0.00 & 0.37 & 0.18 \\
\hline \multirow[t]{2}{*}{ ARGENTINA } & CLASE & $\mathrm{M}$ & $M$ & $\mathrm{M}$ & $\mathbf{N}$ & $\mathrm{N}$ & $\mathrm{M}$ & $\mathrm{M}$ & $\mathbf{N}$ & B & $\mathrm{M}$ & $\mathrm{M}$ & $M$ \\
\hline & VTR-EMN & 1.60 & 12.60 & 0.19 & 1.90 & 1.26 & 1.64 & 0.80 & 0.00 & 0.31 & 0.00 & 0.64 & 0.32 \\
\hline \multirow[t]{2}{*}{ BRASIL } & CLASE & M & $\mathbf{N}$ & $M$ & $\mathrm{~N}$ & C & C & $M$ & $M$ & B & $M$ & B & $M$ \\
\hline & VTR-EMN & 1.91 & 5.73 & 0.00 & 0.00 & 1.08 & 0.00 & 0.00 & 0.00 & 0.83 & 0.00 & 0.00 & 0.00 \\
\hline \multirow[t]{2}{*}{ CHILE } & CLASE & $\mathrm{N}$ & $\mathbf{N}$ & $\mathrm{M}$ & $\mathrm{M}$ & C & $\mathrm{M}$ & $M$ & $\mathrm{~N}$ & B & $\mathrm{M}$ & $\mathrm{M}$ & $M$ \\
\hline & VTR-EMN & 3.34 & 10.02 & 0.00 & 0.00 & 0.00 & 0.00 & 0.00 & 0.00 & 0.29 & 0.00 & 0.00 & 0.67 \\
\hline \multirow[t]{2}{*}{ COLOMBIA } & CLASE & $\mathbf{N}$ & $\mathbf{N}$ & M & $M$ & $M$ & M & $M$ & $M$ & M & $M$ & B & M \\
\hline & VTR-EMN & 0.00 & 0.00 & 0.00 & 0.00 & 0.00 & 0.00 & 0.00 & 0.00 & 0.00 & 0.00 & 0.00 & 0.00 \\
\hline \multirow[t]{2}{*}{ CUBA } & CLASE & $M$ & $M$ & $M$ & $M$ & $M$ & $M$ & $M$ & $M$ & B & $M$ & $M$ & $M$ \\
\hline & VTR-EMN & 0.55 & 2.46 & 0.14 & 0.00 & 0.31 & 0.73 & 0.79 & 0.00 & 0.38 & 0.00 & 0.94 & 0.71 \\
\hline \multirow[t]{2}{*}{ MEXICO } & CLASE & $M$ & $\mathrm{~N}$ & M & $M$ & $M$ & $M$ & $M$ & $M$ & B & $M$ & B & M \\
\hline & VTR-EMN & 0.00 & 0.00 & 1.78 & 0.00 & 0.64 & 1.00 & 0.00 & 0.00 & 0.30 & 0.00 & 1.29 & 1.81 \\
\hline \multirow[t]{2}{*}{ SALVADOR } & CLASE & M & $M$ & C & $M$ & $\mathbf{M}$ & $\mathbf{M}$ & $\mathrm{M}$ & $M$ & B & $M$ & B & C \\
\hline & VTR-EMN & 2.95 & 0.00 & 1.54 & 21.15 & 0.00 & 0.43 & 1.41 & 0.00 & 0.34 & 0.00 & 0.32 & 0.20 \\
\hline \multirow[t]{2}{*}{ VENEZUELA } & CLASE & M & M & M & C & M & M & M & M & B & M & M & M \\
\hline & VTR-EMN & 1.05 & 0.63 & 0.44 & 0.32 & 0.30 & 0.14 & 0.83 & 0.00 & 1.04 & 0.50 & 1.35 & 1.40 \\
\hline \multirow[t]{2}{*}{ CHINA } & CLASE & M & $M$ & M & $M$ & $M$ & $M$ & $M$ & M & B & $M$ & B & B \\
\hline & VTR-EMN & 0.45 & 0.00 & 0.53 & 0.00 & 0.70 & 0.94 & 0.97 & 0.00 & 0.75 & 0.00 & 1.86 & 0.76 \\
\hline \multirow[t]{2}{*}{ COREA SUR } & CLASE & M & $M$ & M & $M$ & $M$ & $M$ & M & $M$ & B & $M$ & B & $C$ \\
\hline & VTR-EMN & 0.71 & 0.71 & 0.87 & 0.00 & 0.13 & 0.31 & 1.02 & 0.00 & 1.28 & 0.00 & 1.43 & 1.75 \\
\hline \multirow[t]{2}{*}{ HONG-KONG } & CLASE & M & M & M & M & M & M & $\mathrm{M}$ & M & B & M & C & B \\
\hline & VTR-EMN & 0.79 & 0.68 & 0.00 & 0.35 & 0.51 & 0.10 & 0.24 & 0.00 & 0.90 & 0.54 & 0.66 & 1.44 \\
\hline \multirow[t]{2}{*}{ INDIA } & CLASE & M & $M$ & M & M & $M$ & $M$ & $M$ & $M$ & B & M & M & B \\
\hline & VTR-EMN & 0.86 & 0.47 & 0.24 & 2.16 & 0.09 & 0.07 & 0.34 & 1.43 & 0.99 & 0.00 & 2.91 & 2.76 \\
\hline \multirow[t]{2}{*}{ SINGAPUR } & CLASE & M & M & M & $\mathbf{N}$ & $M$ & $M$ & M & $\mathrm{N}$ & B & $M$ & C & C \\
\hline & VTR-EMN & 0.87 & 0.00 & 0.86 & 0.00 & 0.49 & 0.43 & 1.39 & 0.00 & 0.82 & 0.00 & 2.21 & 1.78 \\
\hline TAIWÁN & CLASE & M & $M$ & C & $M$ & $M$ & M & $\mathrm{N}$ & $M$ & B & $M$ & C & B \\
\hline
\end{tabular}

Fuente: "Espace Bulletin 1978-02" y actualización para 2003, EPO y elaboración propia. 


\section{Conclusiones y reflexiones finales}

Este trabajo tenía como objetivo contrastar si las EMN ejercían un papel activo en la formación de competencias tecnológicas nacionales de países seguidores y, de ser así, identificar la existencia de diferentes patrones de inserción en la formación de competencias tecnológicas nacionales. Finalmente, y de acuerdo con cada forma de inserción, el trabajo pretendía arrojar alguna luz sobre la relevancia del las EMN en sus procesos de captura.

Para ello, el trabajo utilizó datos de patentes de la European Patent Office para 14 países seguidores, ocho latinoamericanos y seis asiáticos, contabilizando el número de patentes cuyos inventores registraban tener residencia en estos países. Fueron utilizados como indicador de especialización la Ventaja Tecnológica Revelada y como indicador de convergencia la Chi-cuadrado.

Las conclusiones extraídas del análisis, de acuerdo con el objetivo, son las siguientes. En primer lugar, las EMN ejercen, sí, un papel activo en la formación de competencias excepto en dos de los países analizados - Cuba y Corea del Sur. En segundo lugar, la forma en que es ejercida esta actividad varía de acuerdo con su importancia relativa respecto de otros agentes sobre el total de patentes. En los países donde la cuota de patentes por agente usuario se concentra en EMN, ellas son las responsables por la formación de competencias. Son los casos de El Salvador y Singapur. Entre estos dos casos extremos, las EMN son relevantes en la composición de todo tipo de competencias en Chile, Colombia y Venezuela; o bien, son relevantes en aquellas competencias donde los países están especializados (Argentina y Brasil) acentuando su especialización; o bien son relevantes en competencias donde los países no están especializados (India y Hong-Kong), ayudando a la diversificación de su base técnica. De otro lado, a pesar de que teóricamente las EMN estarían más interesadas en la duplicación de competencias que en el aprovechamiento de competencias locales, la inserción de las EMN en países seguidores pasa por el aprovechamiento, en mayor o menor medida, de algunas de sus ventajas locales (competencias centrales y nicho).

Finalmente, y respondiendo al tercer objetivo, aunque el artículo no analiza la importancia de la inserción de las EMN en los procesos de convergencia registrados en el pasado, vistos los resultados y atendiendo a las ideas expuestas por la literatura, una conclusión puede ser extraída para los cuatro 
países donde el patrón de inserción se muestra más claro. La acción de las EMN en la India y Hong-Kong favorece la diversificación de la base técnica en campos técnicos de elevada permeabilidad, oportunidades tecnológicas medias y vinculados a actividades productivas dinámicas, lo que debe conducir en el futuro a una aceleración de los ritmos de convergencia tecnológica. Sin embargo, la acción de las EMN en Brasil y Argentina favorece la sobreespecialización que estos países mantienen en campos técnicos de baja oportunidad tecnológica, poco permeables y vinculados a actividades productivas poco dinámicas, lo que podría comprometer en el futuro sus ritmos de convergencia tecnológica.

\section{Referencias bibliográficas}

Archibugi, D.; Pianta, M., "The technological specialization of advanced countries", Dordrecht, Kluwer Academic, 1992.

Cantwell, J., "The globalisation of technology: what remains of the product cycle model?", Cambridge Journal of Economics, v.19, p.155-174, 1995.

Cantwell, J.; Janne, O., "Technological globalisation and innovative centres: the role of corportate technological leadership and locational hierarchy”, Research Policy, v.28, p.119-144, 1999.

Gerybadze A.; Reger, G., "Globalisation of R\&D: recent changes in the measurement of innovation in transnational corporations", Research Policy, v.28, p.251274, 1999.

Kuemmerle, W., "Foreign Direct Investment in Industrial Research in the Pharmaceutical and Electronics Industries. Results from a Survey of Multinational Firms", Research Policy, v.28, p.179-193, 1996.

Malerba F.; Montobbio, F., "Exploring factors affecting international technological specialization", Journal of Evolutionary Economics, v.13, p.411-434, 2003. 
Pavitt, K., "Uses and abuses of patents statistics", en: Van Raan, A.F.J., Handbook of quantitative studies of science and technology, North Holland: Elsevier Science Publishers, B.V., p.509-536, 1988.

Patel, P.; Vega, M., "Patterns of internationalisation of corporate technology: location vs. home country advantages", Research Policy, v.28. p.145-155, 1999.

Patel, P.; Pavitt, K., "Technological competencies in the world's largest firms: characteristics, constraints and scope for managerial choice", STEEP Discussion Paper, $\mathrm{n}^{\circ}$ 13, SRPU, University of Sussex, 1993.

Perez, C.; Soete, L., "Catching up in technology", en: Dosi, G. et al (eds.) Technical Change and Economic Theory, London: Pinter Publishers, p.458-479, 1988.

Pianta, M.; Meliciani, V., "Technological specialization and economic performance in OECD countries", Technology Analysis \& Strategic Management, v.8, n.2, p.157-174, 1996.

Rocha, C.F.; Urraca, A., "Internacionalização da P\&D das empresas transnacionais. Especialização produtiva nacional e competências tecnológicas”, Economia e Sociedade, v.11, n.1, p.165-183, 2002.

Urraca, A., "Empresas multinacionales, especialización tecnológica y convergencia en países catching-up: América Latina”, Economia e Sociedade, v.14, n.1, p.123, 2005.

Wortmann, M., "Multinationals and the internationalization of R\&D: New developments in German companies", Research Policy, v.19, p.175-183, 1990.

Zander, I., "The evolution of technological capabilities in the multinational corporation - dispersion, duplication and potential advantages from multinationality”, Research Policy, v.27, p.17-35, 1998.

"How Do You Mean 'Global'? An Empirical Investigation of Innovation Networks in the Multinational Corporation". Research Policy, v.28, p.195213, 1999. 